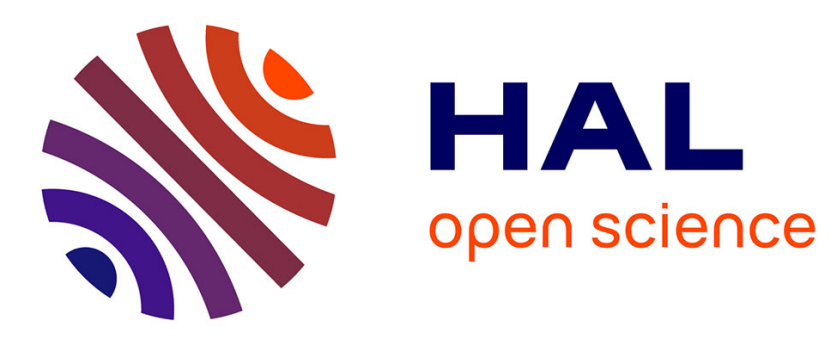

\title{
L'identification inconsciente au fantôme
}

Sylvie Le Poulichet

\section{To cite this version:}

Sylvie Le Poulichet. L'identification inconsciente au fantôme. Cliniques méditerranéennes, 2012, L'enfant et ses fantômes, 2 (86), pp.21-32. 10.3917/cm.086.0021 . hal-01514594

\author{
HAL Id: hal-01514594 \\ https://hal.science/hal-01514594
}

Submitted on 28 Jun 2017

HAL is a multi-disciplinary open access archive for the deposit and dissemination of scientific research documents, whether they are published or not. The documents may come from teaching and research institutions in France or abroad, or from public or private research centers.
L'archive ouverte pluridisciplinaire HAL, est destinée au dépôt et à la diffusion de documents scientifiques de niveau recherche, publiés ou non, émanant des établissements d'enseignement et de recherche français ou étrangers, des laboratoires publics ou privés. 


\section{Sylvie Le Poulichet}

\section{L'identification inconsciente au fantôme}

Dans la nouvelle intitulée La redevance du fantôme ${ }^{1}$, Henry James présente une fiction énigmatique recélant quelques intuitions relatives au thème du fantôme. Il est question dans cette nouvelle de la redevance payée par une fille à son propre père, après que ce dernier l'a en quelque sorte " tuée » par ses paroles de condamnation. Orpheline de mère, cette fille, qui tient désormais le rôle d'un fantôme, obtient le droit d'occuper la maison pour la hanter en payant une redevance, renonçant du même coup à sa propre vie et restant à sa manière possédée par son père. Jusqu'au jour où se manifeste sa croyance en un autre fantôme qui vient la déloger de sa place. Qui est le véritable fantôme? Ne serait-il pas un autre que celui qui a été dans un premier temps supposé ?

Dans le champ de la psychanalyse, que serait une identification inconsciente au fantôme ? S'agirait-il d'une «identification mélancolique » au sens freudien ou d'une autre forme d'identification?

\section{CRYPTE ET MÉLANCOLIE}

Rappelons qu'en 1917, dans « Deuil et mélancolie », Freud annonçait d'emblée que la mélancolie « se présente sous des formes cliniques diverses dont il n'est pas certain qu'on puisse les rassembler en une unité ${ }^{2} »$; de plus, il ajoutait que son matériel se limitait à un petit nombre de cas. Il disait alors abandonner toute prétention à ce que les résultats de son travail « aient une validité universelle », ces résultats ne concernant qu'un groupe restreint.

La mélancolie témoigne pour Freud d'une «perte de l'objet qui est soustraite à la conscience », " le malade sachant sans doute qui il a perdu mais

Sylvie Le Poulichet, psychanalyste, professeure de psychopathologie à l'université Paris 7-Denis Diderot ; 4 rue Dareau F-75014 Paris - lepoulichet.sylvie@neuf.fr

1. H. James (1876), La redevance du fantôme, Paris, Librairie générale française, 1988.

2. S. Freud (1917), « Deuil et mélancolie », dans Métapsychologie, Paris, Gallimard, 1968, p. 147. 
non $c e$ qu'il a perdu en cette personne $^{3} »$. Et Freud postule une identification inconsciente du moi avec l'objet abandonné, l'amour d'objet se trouvant désormais remplacé par cette identification. Cependant, face à ce processus assez répandu qui peut caractériser tout deuil difficile, la spécificité du " tableau mélancolique » réside dans le fait que se déchaîne une ambivalence par rapport à l'objet, cette dernière se retournant contre le moi. Du même coup, une partie du moi, que Freud nommera en 1923 le «surmoi », s'oppose à l'autre partie du moi identifiée à l'objet et l'attaque de manière "sadique ». De là découle la "diminution du sentiment d'estime de soi qui se manifeste en auto-reproches et auto-injures ${ }^{4}$ ", s'ajoutant à la dépression, à la suspension de l'intérêt pour le monde extérieur, à l'inhibition de toute activité, ainsi qu'à la perte de la capacité d'aimer.

Il apparaît bien que l'identification inconsciente du moi avec l'objet perdu peut aussi avoir d'autres destins pathologiques que celui de la mélancolie, notamment dans la névrose obsessionnelle et dans certaines phobies. Quant à l'identification inconsciente à un fantôme (dont Freud ne parle pas), elle présente un destin que l'on doit davantage explorer car, si elle témoigne également d'une « perte de l'objet qui est soustraite à la conscience » et d'une identification inconsciente à cet objet, j'émets l'hypothèse qu'en ce cas le moi ignore fondamentalement à la fois qui il a perdu et $c e q^{\prime}{ }^{\prime} i l$ a perdu en cette personne.

Les travaux de Nicolas Abraham ont eu le mérite d'éclairer des éléments cruciaux, en particulier lorsqu'il décrit l'action d'un fantôme sur la génération suivante et qu'il note : « Le fantôme qui revient hanter est le témoignage de l'existence d'un mort enterré dans l'autre ${ }^{5}$. » Néanmoins, sa théorie de la « crypte intrapsychique » suscite certaines interrogations au regard des expériences analytiques. Car ce qu'il nomme "l'identification endocryptique » serait le résultat d'une "inclusion » qui, paradoxalement, ne concernerait pas le fantasme du patient. Comme si le sujet avait directement hérité d'un fantôme et que ce sujet ne saurait aucunement "être mis en cause ", selon l'expression de Nicolas Abraham, dès lors qu'il se trouve possédé par un " corps étranger » radicalement " hétérogène ${ }^{6}$ ». Et, ajoute-t-il, par l'effet de la construction dans l'analyse, toute cette histoire pourrait se trouver " déconstruite ", "sans que le sujet ait eu l'impression d'avoir été lui-même analysé en tant que sujet ${ }^{7} »$. Il paraît difficile de suivre Nicolas Abraham sur ce point précis, qui semble annuler l'expérience et la réflexion psycha-

3. Ibid., p. 151.

4. Ibid., p. 148.

5. N. Abraham, L'écorce et le noyau, Paris, Aubier-Flammarion, 1978, p. 431.

6. Ibid., p. 430-431.

7. Ibid., p. 430. Albert Ciccone a présenté une importante critique de ces conceptions dans son ouvrage La transmission psychique inconsciente, Paris, Dunod, 1999, p. 79-104. 
nalytiques relatives aux processus identificatoires et fantasmatiques consécutifs à des transmissions traumatiques. En effet, les anciennes discussions portant sur la distinction entre les étiologies purement traumatiques et celles purement fantasmatiques semblent le plus souvent dépassées, puisque les transmissions traumatiques génèrent elles-mêmes des identifications inconscientes et des constructions fantasmatiques. Comment suffirait-il à l'analyste de reconstituer et de nommer l'héritage d'un fantôme pour qu'un patient termine sa cure sans avoir été confronté à l'analyse de ses propres processus inconscients, ni au travail de transformation de son angoisse par rapport à ce fantôme ? Je reviendrai plus en détail sur ces questionnements, que je tenterai de soumettre à l'épreuve de cette clinique où, précisément, selon la judicieuse proposition de Nicolas Abraham, « l'apparition du fantôme indiquerait donc les effets sur le descendant de ce qui avait eu, pour le parent, valeur de catastrophe narcissique ${ }^{8} »$.

\section{LE PACTE ET LA MISSION}

L'analysante dont il sera ici question eut un père qui avait insisté pour lui donner à sa naissance un unique prénom de son choix. Ce prénom rappelait la mort de la mère de cet homme, mère morte en accouchant de lui. Toute jeune, la future analysante avait appris qu'à la suite du décès de cette grandmère réputée "si gentille et si fragile ", son père avait été confié à différentes femmes de la famille, mais n'avait pas eu durablement de place légitime chez son propre père, qui s'était rapidement remarié avec une femme rejetant l'enfant.

La jeune future analysante, que je désignerai par la lettre « $V$ », n'avait appris tout cela qu'à travers quelques confidences chuchotées par sa propre mère, car son père n'avait jamais évoqué un seul élément de sa propre histoire et, de surcroît, n'adressait que très rarement la parole à sa fille, lui qui semblait flotter dans la vie comme une ombre. V. se souvient que lorsqu'elle était très jeune, sa mère lui avait discrètement et rapidement désigné de loin, dans une pièce de la maison du grand-père paternel, un cadre contenant une photographie de la morte. Mais V. n'avait rien vu car la lumière frappait le verre recouvrant la photographie. En somme, il s'était produit une apparition de « rien » ou d'un éclat de lumière dans un cadre, puis une disparition définitive, la photographie ayant été ensuite retirée. V. connut son père le plus souvent silencieux, le regard presque toujours perdu dans le lointain, ne s'animant qu'à l'occasion de ses activités de défense des droits des ouvriers étrangers. Parallèlement, ce père ne cessait de se mettre

8. Ibid., p. 430. 
en danger à travers de nombreux accidents. En outre, la mère de V. dévalorisait de plus en plus son époux et multipliait les reproches à son égard. Elle ne protégeait d'ailleurs pas davantage sa propre fille.

$\mathrm{C}^{\prime}$ est seulement au cours de son analyse que $\mathrm{V}$. commence à entrevoir certains liens entre différents éléments qui ne résonnaient aucunement entre eux auparavant. Notamment cet épisode qu'elle n'avait rattaché à rien : durant plusieurs années de son enfance, elle s'était identifiée à l'héroïne d'une série de livres de la "Bibliothèque rose ", relatant les exploits d'une fillette justicière qui partait en mission durant la nuit, déguisée et masquée. V. s'était alors elle-même confectionné un semblable déguisement et improvisait des missions nocturnes. Bien plus tard, elle éprouva en séance une vive émotion en réalisant que le prénom porté le jour par l'héroïne des romans était identique à celui de sa propre grand-mère décédée en couches.

Un autre élément sortit de l'oubli, issu des nombreux souvenirs d'accidents de voiture qu'elle avait connus lorsque son père conduisait. Ce dernier la contraignait en effet pour la conduire en différents lieux. Un jour, lorsque le véhicule longeait le haut mur bordant une propriété privée et qu'un grand virage bien connu se présentait, son père ne touchait plus le volant. Il semblait qu'il n'allait pas prendre le virage, mais plutôt lancer le véhicule contre le mur. V., alors âgée de 9 ans, se précipita depuis le siège arrière de la voiture pour saisir et tourner le volant. Aucun commentaire ne s'ensuivit : l'un et l'autre seraient restés muets. De plus, V. n'a ensuite jamais parlé à quiconque de cet épisode, comme si une partie d'elle savait qu'un pacte mystérieux et secret la liait ainsi à son père. Quant au léger sourire du père au moment où il avait lâché le volant, il semblait maintenant à l'analysante qu'il pouvait traduire une pensée comme : "Qu'il serait merveilleux de mourir ensemble soudain, tous les deux!»

Lorsque $\mathrm{V}$. devint adolescente, elle apprit que son père était gravement malade, que ses jours étaient en danger. C'est à ce moment-là qu'elle bascula dans un épisode délirant à bas bruit durant plusieurs semaines. À la suite d'une prise de cannabis, elle fut en effet envahie par la certitude qu'elle venait de mourir, mais que personne ne le savait. Elle gardait secrète sa conviction et continuait de fréquenter son lycée d'une manière étrange et détachée. Selon ses dires, un sentiment de toute-puissance l'habitait constamment et elle pensait : « Les autres ne savent pas que je suis morte et que je reviens juste quelque temps sur terre pour accomplir une mission. » La mission consistait essentiellement à empêcher le décès du père.

Quelque temps après la fin de cet épisode délirant, que personne dans son entourage n'avait clairement perçu, elle se retrouva très angoissée et demanda à consulter un psychothérapeute. Elle ne cessa de pleurer durant ses séances hebdomadaires dans un centre de consultation pour adolescents, 
où elle était reçue par une thérapeute totalement silencieuse. V. dit qu'en sortant de ses séances, elle errait près de la Seine et songeait sans cesse à se noyer. Trois ans plus tard, son père décède. V. ne se remet pas de cette mort, même si elle réussit des études qui la mènent à exercer une profession rappelant son ancien personnage de justicière.

Ce n'est que dix ans après la mort de son père qu'elle vient me consulter et relate toute cette histoire qui n'apparaît en fait qu'à travers des bribes éparpillées au sein du récit bien plus vaste de son enfance et de sa vie d'adulte fort mouvementée, où elle ne cesse de se sentir en danger et où elle a régulièrement l'impression qu'une mort imminente la menace. Il s'agissait bien là de deux manifestations pouvant résulter d'identifications au père et à la grand-mère.

\section{FONCTIONS DU RÊVE ET DU DÉLIRE}

DANS LA TRAVERSÉE DE L'IDENTIFICATION AU FANTÔME

C'est un rêve particulier qui semble avoir précipité cette fois sa demande $\mathrm{d}$ 'analyse : l'unique rêve où se produisit une apparition de sa grand-mère décédée en couches. V. s'y trouvait comme paralysée, allongée sur un lit, tandis que surgissait au pied du lit cette femme aux caractéristiques apparemment opposées à celles de sa grand-mère. Dans le rêve, il s'agissait d'une forte femme volontaire d'origine asiatique, vêtue d'un kimono, qui lui déclarait d'un ton vif : « Me voilà enfin, $c^{\prime}$ est maintenant que nous avons rendezvous! » Un certain décollement ou une ouverture pouvait-elle alors s'opérer inconsciemment et laisser émerger une demande d'analyse adressée à une femme, dès lors que la morte apparaissait enfin en étrangère, radicalement différente de V. ? De plus, un enjeu crucial apparaissait d'emblée à travers ce rêve inaugural puisque les places semblaient inversées dans le rêve : paralysée et allongée sur un lit, V. n'occupait-elle pas partiellement la place de la morte ? Et néanmoins, la présence du coussin de kimono sur le corps de la femme ne représentait-elle pas une allusion à l'ancienne porteuse d'enfant ?

Cependant, de manière consciente, c'était pour parler du fantôme de son père que $\mathrm{V}$. vint me consulter. Et il apparut que $\mathrm{V}$. avait développé une identification ambivalente au père tellement aimé et redouté. Je n'évoquerai pas ici en détail la manière dont l'image de ce père venait la hanter, mais je mentionnerai à ce propos un moment décisif du début de la cure. L'envahissement par la figure du père était allé jusqu'à provoquer, lors d'une séance, un moment de type hallucinatoire où l'analysante percevait le visage du père s'approchant de plus en plus du sien, comme s'ils allaient se confondre. Et j'étais intervenue juste avant que les deux images ne coïncident, afin de les séparer, à l'instant où l'analysante au comble de l'angoisse arrivait à arti- 
culer quelques mots pour m'appeler au secours. À ce moment avait donc surgi dans l'espace de la séance un véritable mouvement de fantôme à travers une formation hallucinatoire. Moment critique s'il en est, recélant néanmoins une capacité métamorphique : au-delà de la terreur éprouvée, il avait pu représenter un événement crucial dans le transfert, appelant la figure d'un tiers analyste qui interrompt le mouvement de l'identification au fantôme et ouvre de nouvelles voies d'analyse du lien au père. Le " moment " (du latin momentum, issu de movimentum et movere) s'entend bien comme un mouvement, en l'occurrence ici mouvement de fantôme. Et ce mouvement ou moment critique, mettant en jeu une crise (crisis) relative à la séparation entre mort et vivant, s'assimilait à un vacillement identificatoire périlleux. Il fallait bien néanmoins qu'arrive dans le transfert un tel mouvement, afin que ses ressorts psychiques trouvent une nouvelle issue, à condition qu'un analyste soit là pour percevoir et intercepter ce mouvement.

Cependant, au-delà du travail autour du mouvement d'union avec le père mort, il apparut que ce lien ne pouvait être véritablement dénoué que par la reconstruction et par l'élaboration du rapport au fantôme de la grandmère, notamment grâce aux liaisons effectuées entre les éléments que j'ai rapportés plus haut.

Comme je l'avais indiqué, V. n'avait auparavant jamais fait le rapprochement entre le prénom de cette grand-mère et le prénom de son héroïne justicière masquée à laquelle elle s'était identifiée. Elle pouvait dire maintenant qu'elle se souvenait avoir toujours veillé en secret sur son père, absorbée par le souci de le protéger. Parallèlement à d'autres mouvements psychiques, elle s'était bien identifiée inconsciemment et précocement à cette grand-mère, ce que révélait avec une grande intensité son épisode délirant à l'adolescence. À ce sujet, je lui proposai cette interprétation : face au danger de la mort de son père, elle était à ce moment-là devenue - à son insu - la mère morte qui revient sur cette terre pour sauver son fils, persuadée de le guérir par son pouvoir magique. J'ajoutai que cette identification inconsciente à sa grand-mère était sûrement déjà présente lorsqu'elle avait banalisé l'épisode où son père aurait souhaité qu'ils meurent ensemble dans un accident de voiture : comme si un pacte inconscient les unissait secrètement et comme si cette " mort ensemble » avait le pouvoir d'effacer ou de corriger la scène première où la grand-mère mourait seule en donnant la vie à son fils. Je précisai qu'une partie d'ellemême avait dû depuis longtemps s'identifier inconsciemment à la mère de son propre père et que cela devait probablement résonner avec certainsfantasmes inconscients de ses parents. En effet, d'autres éléments troublants s'étaient produits, en plus du choix du prénom de V. Il s'agissait en particulier d'un lapsus de sa mère qui lui avait déclaré : «Tu le sais bien, cela s'est passé juste un an avant ta mort! », alors qu'elle voulait évoquer la naissance de V. À cela 
s'ajoutait la parole que lui avaient adressée ses parents, un an avant la mort du père, lorsque $\mathrm{V}$. avait décidé de quitter le domicile familial afin de poursuivre ses études dans une autre ville: "Puisque tu le veux, va-t'en, mais nous te coupons les vivres et tu n'es plus notre fille ! » Parole insensée prenant valeur de désengendrement de leur fille.

Au sein du vaste puzzle que présentait son passé, il avait fallu extraire et mettre en lien ces différentes pièces, ces morceaux auparavant isolés, afin que le sens de son histoire apparaisse et que $\mathrm{V}$. puisse s'en saisir à la première personne du singulier. S'identifier au fantôme de la grand-mère et devenir ainsi mère de son propre père : cette formule de l'interprétation avait d'abord sidéré l'analysante qui s'en était néanmoins rapidement emparée, en se dégageant peu à peu de l'énorme charge qui avait depuis si longtemps pesé sur elle et qui l'avait partiellement inscrite du côté des morts, ou dans " l'aire des revenants ${ }^{9} »$. Provoquant la dissolution d'un fantasme crucial, ce mouvement dans l'analyse impliquait maintenant la traversée d'un passage angoissant mettant en jeu la question fondamentale : «Mais qui suis-je alors ? » La recomposition des identifications et des investissements d'objet pouvait désormais s'effectuer, d'autant plus que l'identification au fantôme était restée partielle et que l'épisode délirant de l'adolescence avait été unique : comme en bien des « cas limites", diverses strates psychiques avaient permis parallèlement le déploiement d'autres potentialités. Plus précisément, deux strates psychiques semblaient s'être superposées dans le devenir de V. : l'une relevant du complexe d'CEdipe, l'autre de processus de déni et de clivage.

Je reviens maintenant au rêve qui avait précipité sa demande d'analyse : je disais plus haut qu'il figurait pour la première fois la grand-mère morte sous les traits d'une étrangère lui donnant un " rendez-vous » et qu'il engageait à ce titre un premier décollement. De fait, la possibilité d'un transfert s'inscrivait dans ce sillage : sans le savoir au départ, l'analyste allait pouvoir donner un corps étranger et vivant à ce fantôme, tout en en délestant peu à peu l'analysante. Occuper la place de celle qui entend et qui veille dans la continuité, mais aussi de celle qui investit, parle et protège d'une certaine façon, c'était là engager une petite révolution dans la distribution des rôles. Finalement, c'est sans doute ce que V. était déjà venue chercher en vain dans sa thérapie à l'adolescence, au moment où - dans son silence et son figement - l'ancienne thérapeute se mettait malencontreusement à la place de la morte muette, se transformant du même coup en une figure

9. Telle que je l'ai définie dans mon ouvrage Les chimères $d u$ corps : il s'agit de cette zone inconsciente où le vivant s'efface au profit du mort, lorsque le " complexe de l'être humain proche " (complexe du Nebenmensch selon Freud) se révèle défaillant. Voir S. Le Poulichet, « Le miroir et l'excorporation des morts ", dans Les chimères du corps. De la somatisation à la création, Paris, Aubier, 2010, p. 103-123. 
surmoïque féroce suscitant une "répétition du traumatisme ${ }^{10}$ ». Cette répétition avait alors provoqué chez $\mathrm{V}$. des impulsions suicidaires, comme si elle se retrouvait cette fois identifiée au père, tous deux tombant sous le coup de l'abandon par la figure d'une grand-mère froide et indifférente, représentée par la thérapeute.

Dans son analyse ultérieure, il apparaissait qu'un tiers maternel et grand-maternel était appelé au cœur du transfert, afin d'inscrire l'analysante dans une double triangulation. En cette cure, il fallait à la fois qu'un substitut de la mère reconnaisse le père et qu'un substitut de la grand-mère apparaisse comme un " porte-parole ${ }^{11}$ » avec lequel V. aurait " rendez-vous », afin de distinguer les places de $V$., de son père et de la grand-mère sur trois générations. Et l'identification au fantôme fut effectivement abolie au cours de cette analyse de V., par l'œuvre de cette double triangulation qui était auparavant restée en souffrance. Il s'agissait également de substituer une loi de présence, de reconnaissance et de parole articulée, à la loi du silence et de la destruction véhiculée par l'histoire familiale. Le fait que l'analyste porte des énoncés identifiants qui réinscrivent du désir dans le vivant dissolvait chez l'analysante une condamnation à se penser comme extra-terrestre et absolument solitaire, vouée au "désaide » et au " désêtre », un tel destin ayant représenté auparavant une évidence totale ou une mission incontournable.

\section{L'EXCORPORATION DU MORT PRÉCÉDANT LE TRAVAIL DE DEUIL}

Si des " effets de fantôme » peuvent se manifester dans le cadre de certains deuils sans impliquer tout le processus ici déployé et si l'identification au fantôme constitue une formation différente de la mélancolie (où la dimension ignorée de la haine à l'égard de l'objet perdu se retourne contre le moi qui, du même coup, s'accable de reproches et perd toute estime de soi), il importe de définir la spécificité de cette identification inconsciente au fantôme.

Il existe en fait de nombreux modes d'identification au fantôme qui ne déclinent pas tout à fait les mêmes devenirs psychiques selon qu'ils mettent en jeu simultanément un membre de la fratrie, un parent ou un grand-parent, voire des embryons disparus au cours d'avortements ou de fausses couches. Et c'est, de plus, le contexte familial tout entier qui permettra ou ne permettra pas à une figure de fantôme d'errer en produisant des moments de dépersonnalisation, des mouvements hallucinatoires ou d'autres symptômes. En effet,

10. Selon la formule de S. Ferenczi dans son article de 1934 « Réflexions sur le traumatisme ", dans CEuvres complètes, tome IV, Paris, 1982, Payot, p. 139-147.

11. Selon l'expression de Piera Aulagnier dans son ouvrage La violence de l'interprétation, Paris, PUF, 1975. 
tout décès d'un proche ou toute disparition d'un fœetus n'engendre pas une identification inconsciente à un fantôme.

La particularité de cette clinique ne peut à mon avis se ramener entièrement aux propositions de Nicolas Abraham, pour qui une « crypte » immobile et hétérogène subsiste à la manière d'un pur corps étranger à l'intérieur d'un sujet, à l'écart de son propre fantasme (cette «identification endocryptique » étant supposée pouvoir se trouver déconstruite en analyse « sans que le sujet ait eu l'impression d'avoir été lui-même analysé en tant que sujet $\left.{ }^{12} »\right)$. En fait, c'est bien dans la mesure où l'analyste reconnaît la réalité de disparitions, de " secrets de famille » et de condamnations implicites ou explicites, que deviennent analysables les formations fantasmatiques de l'analysant, s'appuyant sur des identifications inconscientes précoces. L'analysant qui n'a pu échapper à une forme d'incorporation du mort ne saurait dans la cure faire l'économie de l'expérience d'une intime " excorporation » du mort, ayant le pouvoir de recomposer ses repères identificatoires et les formules de son désir, à la suite d'une traversée de l'angoisse inhérente à la modification du fantasme, cette traversée s'accompagnant souvent d'épisodes passagers de dépersonnalisation ou de sensations fugaces de fragmentation corporelle, ou encore de mouvements hallucinatoires. Une telle « excorporation » du ou des morts ne s'effectue pas à la faveur d'une simple reconstruction historique : $c^{\prime}$ est bien une forme d'interprétation par l'analyste, accueillant les mouvements de fantômes, qui peut déclencher ce processus et dissoudre un mode de déni et de clivage. La notion d'excorporation du mort diffère de celle du deuil, car dans cette clinique, c'est bien la première qui rend possible le deuil en abolissant le déni et le clivage. Une telle mutation ne peut s'accomplir qu'à l'instar de la traversée d'événements psychiques se produisant dans le rêve et le transfert, provoquant un ou des moments critiques qui permettront de recomposer les figures du corps et des origines de l'analysant ${ }^{13}$. Et $\mathrm{c}^{\prime}$ est seulement à la suite de ces processus que pourra s'accomplir un «travail de deuil », en son sens freudien, au cours duquel « l'accomplissement en détail

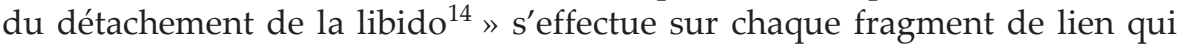
reliait le sujet et l'objet.

Si l'œuvre d'une déliaison par la pulsion de mort peut se manifester en cette clinique, ce n'est pas parce que le sujet se trouve simplement, selon l'expression de Nicolas Abraham, " possédé par l'inconscient d'un autre ». La séduction littéraire exercée par le thème du fantôme ne doit pas ici congédier

12. N. Abraham, op. cit., p. 430.

13. En une autre cure, dont j'ai ailleurs relaté de longues séquences, cette excorporation avait donné lieu lors d'une séance à un épisode quasi hallucinatoire de fragmentation du corps, avant que ne se produise lors de la séance suivante une recomposition du désir et de l'unité corporelle. Voir « Le miroir et l'excorporation des morts », op. cit.

14. S. Freud (1917), «Deuil et mélancolie», op. cit., p. 170. 
la métapsychologie. Un inconscient ne saurait être possédé par celui d'un autre, même si des transmissions inconscientes ont eu lieu, participant aux motifs d'une composition psychique singulière chez le descendant. La théorisation de Nicolas Abraham aurait peut-être été influencée par la particularité des épisodes délirants dans cette clinique, lorsqu'ils se présentent. Car ils ont souvent une forme bien délimitée et ne signent pas nécessairement un envahissement du sujet par la psychose.

On ne pourrait dire non plus que le sujet a seulement eu recours au refoulement au sein d'une organisation psychique purement névrotique. Néanmoins, les enjeux névrotiques sont souvent présents en cette clinique, côtoyant des identifications clivées dont la puissance peut temporairement provoquer une " perte de la réalité ${ }^{15}$ " pour laisser éclore un délire, en tant que " tentative de guérison ${ }^{16}$ » de soi-même et de l'autre (même si ces deux tentatives de guérison n'opèrent pas sur le même plan). Il s'agirait là d'un statut particulier de la tentative de guérison portée par un délire résultant d'une identification inconsciente au fantôme, car se présenteraient simultanément une tentative de reconstruction d'un morceau de réalité où le sujet investirait un lien et une tentative de reconstruction de la réalité d'un autre menacé.

\section{CONCLUSION}

Quelle serait finalement la spécificité de l'identification inconsciente au fantôme ? On a vu, à travers le cas clinique présenté, que l'analysante était en quelque sorte passée par-dessus le père pour s'identifier à la grand-mère morte en lui donnant naissance. L'analyse aurait été bien différente si la fille avait seulement développé une identification ambivalente au père aimé et redouté, ainsi qu'à la figure du père rejeté ou toujours menacé de mort, ce dernier représentant lui-même l'objet œdipien. Mais la cure a montré que seule l'analyse de l'identification au fantôme de la grand-mère pouvait durablement apporter un changement dans l'organisation psychique et permettre enfin que s'accomplisse le deuil du père.

Comme en d'autres cas, s'exprime ici une spécificité clinique et théorique de l'identification inconsciente au fantôme : ce n'est pas seulement au parent proche, connu et décédé que le sujet s'identifie, mais aussi à l'une des personnes qui ont engendré ce parent. Le déni porte alors plus particulière-

15. Selon l'expression de Freud, dans son texte « La perte de la réalité dans la névrose et la psychose » (1924), dans Névrose, psychose et perversion, Paris, PUF, 1973, p. 299-303.

16. La conception du délire pouvant représenter une tentative de guérison se trouve mentionnée par Freud dès 1911, dans son texte sur le président Schreber (S. Freud, Cinq psychanalyses, Paris, PUF, 1954, p. 263-324). 
ment sur cette dernière identification à la personne disparue qui n'a le plus souvent jamais été connue, et l'on peut dire également que ce déni porte sur la succession de l'ordre des générations. $C^{\prime}$ est à cet endroit que se manifeste la véritable identification inconsciente au fantôme, clivée, qui implique un renversement de l'ordre des générations, tel un " devenir la mère de son propre père ».

On rencontre aussi cette configuration dans des cures d'enfants où, par exemple, l'analyse de l'identification de l'enfant à un fœtus mort dans le ventre de la mère ne suffit pas toujours à faire disparaître l'angoisse et les symptômes, ces derniers ne cédant que grâce au repérage parallèle d'une autre identification inconsciente de l'enfant (par exemple au père décédé de sa mère), ces processus ne pouvant prendre toute leur dimension que dans le cadre d'une relation de type incestuel avec la mère en cet exemple.

Bien souvent, il faut au moins deux figures de morts, de générations différentes, pour produire une véritable identification inconsciente au fantôme, toujours dans un contexte où la triangulation œdipienne se trouve mise à mal.

\section{BIBLIOGRAPHIE}

Abraham, N. 1978. L'écorce et le noyau, Paris, Aubier-Flammarion.

AulagnieR, P. 1975. La violence de l'interprétation, Paris, PUF.

CicCONE, A. 1999. La transmission psychique inconsciente, Paris, Dunod.

FERENCZI, S. 1934. «Réflexions sur le traumatisme », dans CEuvres complètes, tome IV, Paris, Payot, 1982.

FREUD, S. 1911. «Remarques psychanalytiques sur l'autobiographie d'un cas de paranoïa (Le Président Schreber) », dans Cinq psychanalyses, Paris, PUF, 1954.

FreUD, S. 1917. « Deuil et mélancolie », dans Métapsychologie, Paris, Gallimard, 1968.

FREUD, S. 1924. «La perte de la réalité dans la névrose et la psychose », dans Névrose, psychose et perversion, Paris, PUF, 1973.

JAMES, H. 1876. La redevance du fantôme, Paris, Librairie générale française, 1988.

Le Poulichet, S. 2010. Les chimères du corps. De la somatisation à la création, Paris,

Aubier.

Résumé

Avec la notion d'identification inconsciente au fantôme, il devient possible d'éclairer les processus à l'œuvre dans une clinique où l'identification à l'objet mort n'engendre pas un tableau mélancolique classique, mais un renversement de l'ordre des générations mettant en jeu un déni et un clivage.

À partir de la clinique analytique, l'auteure montre qu'il faut souvent au moins deux morts de générations différentes pour produire une véritable identification 
inconsciente au fantôme, dans un contexte où la triangulation œdipienne demeure défaillante. Des épisodes délirants passagers révèlent souvent cette identification qui pourra donner lieu dans la cure à une excorporation des morts impliquant parfois un moment de fragmentation corporelle préalable au travail de deuil.

Mots-clés

Le délire comme tentative de guérison, excorporation des morts, indentification inconsciente à l'ancêtre disparu, mélancolie, ordre des générations inversé.

\section{THE UNCONSCIOUS IDENTIFICATION TO THE GHOST}

\section{Summary}

The concept of unconscious identification to the ghost enables us to clarify the processes at work in a situation in which identification to the dead object does not generate a classical melancholic clinical picture, but instead a reversal in the order of generations, denial and splitting of the ego being at stake. From the clinics of psychoanalysis, the author demonstrates that there must be very often two deaths of successive generations to produce an unconscious identification to the ghost, in a context in which oedipal triangulation fails. Episodes of transitory delusion may often reveal this identification, which could, in the course of the treatment, give way to an excorporation of the dead, implying sometimes a moment of bodily breakdown prior to the mourning.

\section{Keywords}

Delusion as an attempt of recovery, excorporation of the dead, unconscious identification to the deceased ancestor, melancholia, reversal in the order of generations. 